\title{
Assessment of DOK7 and SPDEF as Potential Epigenetic Biomarkers in Whole Blood of Patients with Gastric Adenocarcinoma and Intestinal Metaplasia
}

\author{
Arash Moradi \\ Department of Medical Biotechnology, National Institute of Genetic Engineering and Biotechnology, \\ Tehran \\ Seyed Ahmad Aleyasin ( $\square$ ahmad.aleyasin1@gmail.com ) \\ Department of Medical Biotechnology, National Institute of Genetic Engineering and Biotechnology, \\ Tehran \\ Masoumeh Rezaii \\ Department of Medical Biotechnology, National Institute of Genetic Engineering and Biotechnology, \\ Tehran
}

\section{Kamal Mohamadian}

Department of Radiation Oncology, Hamadan University of Medical Sciences, Mahdieh center, Hamadan

Aida Alizamir

Department of Pathology, Hamadan University of Medical Sciences, Hamadan

Behrouz Afshar

Department of Internal Medicine, Hamadan University of Medical Sciences, Hamadan

\section{Research Article}

Keywords: gastric cancer, DOK7, SPDEF, epi-biomarkers

Posted Date: February 8th, 2021

DOI: https://doi.org/10.21203/rs.3.rs-156439/v1

License: (c) (1) This work is licensed under a Creative Commons Attribution 4.0 International License. Read Full License 


\section{Abstract}

\section{Background}

Gastric cancer (GC) is the fifth most prevalent cancer, and intestinal metaplasia (IM) is generally considered a precancerous lesion in the gastric carcinogenesis cascade. DNA methylation is one of the most deeply studied epigenetic modifications. Numerous studies have been shown that aberrant methylation of DOK7, an adaptor protein, and SPDEF, a transcription factor, correlates with carcinogenesis. This study aimed to investigate DNA methylation of DOK7 and SPDEF in the whole blood specimens obtained from patients with IM and GC and normal individual controls to examine the possible implication of epigenetic biomarker for differential diagnosis GC from IM.

\section{Material and Methods}

Bioinformatic analysis, differentially methylated regions (DMR) enrichment analysis, was conducted to detect appropriate epigenetic regions in DOK7 and SPDEF CpGs Island. The methylation of selected CPG regions of DOK7 and SPDEF was assayed on 95 blood samples, including $30 \mathrm{IM}$ patients, $30 \mathrm{GC}$ patients, and 35 normal controls individuals. After DNA extraction, MSRE-PCR was utilized to evaluate the loci methylation level, followed by real-time MSRE-PCR to quantify the region's methylation status.

\section{Results}

Our analysis indicated that DOK7 and SPDEF have significant hypermethylation in GC and IM versus the normal specimens. Significant methylation changes were observed for Dok7 between IM ( $p=0.03)$, GC ( $p$ $<0.001$ ) cases compared to normal controls. For SPDEF significant hypermethylation results obtained, for GC $(p<0.001)$ and IM $(p=0.03)$ cases in comparison to normal controls. Sensitivity and specificity for DOK7 as DNA epigenetic biomarker diagnostic of gastric cancer test were determined by ROC statistical analysis revealed high intermediate sensitivity (73.33\%) and high specificity ( $97.14 \%)$ methylation changes with $p<0.001$. For SPDEF DNA methylation test as GC biomarker by ROC statistical analysis revealed lower sensitivity (36.67\%) but higher specificity $(97.14 \%)$ with $p<0.001$.

\section{Conclusions}

These results suggest that the change in the methylation of DOK7 (AUC $=0.9495)$ and SPDEF (AUC = 0.8419 ) is a promising epi-biomarker. For the first time, this study shows blood-based biomarkers DOK7 and SPDEF genes are powerful epi-biomarkers and provide insights into gastric cancer pathogenesis and diagnosis.

\section{Background}

Gastric Cancer, an age-related disease, is one of the most common and fatal cancer worldwide. GC incidence is highly dependent on region and culture and is observed more in men. It accounts for 783,000 deaths each year, as the third most deadly cancer worldwide [1]. The majority of GCs are associated with 
infectious agents, including the bacterium Helicobacter pylori and Epstein-Barr virus (EBV). Injury to the stomach's epithelial layer and inflammation, raised by Helicobacter pylori, can increase gastric stem cells' proliferation [2]. Inflammation also initiates the reprogramming and proliferation of the chief cells into the intestinal cell lineage by epigenetics alterations. Metaplasia refers to representing a normal cell lineage in a tissue where it is not typically found.

Numerous challenges in the gastric cancer study's field remained because of intratumoral, intrapatient, and interpatient heterogeneity in gastric cancer. The first challenge is to overcome gastric cancer incidence high rate through ongoing attention to improved public health and develop precise and less invasive tools and methods for early detection. Eventually, the major challenge is translating recent discoveries in molecular biology to effective treatment for patients with gastric cancer. Therefore, precancerous lesion follow-up is advisable for secondary gastric cancer prevention [3,4].

Therefore, precancerous lesion follow-up is advisable for secondary gastric cancer prevention. Intestinaltype gastric cancer development is preceded by the development of metaplastic cell lineages in the gastric mucosa [5]. The IM prevalence rate is dramatically higher in patients with $\mathrm{H}$. pylori infection. Patients with IM are at a higher risk of malignancy emergence, especially for incomplete IM and IM in the corpus [6]. In most cases, metaplastic lineages are characterized by mucus secretion [7].

Gastric carcinogenesis involves the gradual accumulation of various genetic and epigenetic alterations, leading to gain-of-function in oncogenes and loss-of-function in tumor suppressor genes. Genetic alterations, such as p53 mutations, CD44 amplifications, and epigenetic alterations, including changes in histone modifications, level of DNA methylation, and non-coding RNA expression, have been occurring in tumorigenesis. DNA methylation is a major mechanism of tumor-related gene inactivation, particularly tumor suppressor genes, in the neoplastic cells [8]. Accurate technologies such as whole-genome microarray analysis, immunohistochemical analysis, and DNA methylation analysis allow us to disclose intestinal metaplasia events leading to carcinogenesis.

The epigenetic biomarker's ability to detect cancer in body fluids, such as serum or gastric washes, represents its noninvasive application advantages. For example, a high prevalence of alterations in the gene methylation, such as TFPI2, RUNX3, p16, and CDH1, is found in gastric cancer patients' serum [9]. Unlike tissue DNA, blood leukocyte DNA can be obtained non-invasively and inexpensively. Thus, methylation changes in blood leukocyte DNA may serve as a potential biomarker for disease diagnosis [10].

Dok7 (Downstream of Kinase 7), a peripheral membrane protein, is a Dok family member. It is a cytoplasmic activator of the muscle-specific kinase (MuSK), involved in neuromuscular junction formation and maintenance [11]. CpG site hypermethylation was observed within the DOK7 promoter region in cancers with epithelial origins, such as breast [12] and lung [13] cancers.

SPDEF (SAM Pointed Domain containing ETS transcription factor) is an ETS family transcription factor reported to playing a role in tumor progression and several cancers' metastasis. In the adult intestines, 
SPDEF is a direct downstream target gene of ATOH1, a master transcription factor that mediates Notchregulated differentiation of the intestinal epithelium [14]. SPDEF targets FoxM1 (Forkhead Box M1) promoter activity, which has been proven to be highly expressed in gastric cancer. Wu and colleagues found that SPDEF was overexpressed at the mRNA and protein level in human gastric cancer species [15].

In this study, two potential epigenetic biomarkers (DOK7 and SPDEF) were assessed in the blood leukocytes of patients with IM and GC and normal controls.

\section{Material And Methods}

\section{Data Acquisition}

The relationship between DMCs of gastric tumors and non-tumoral tissue have been checked by downloading microarray data, including non-tumoral and gastric cancerous tissues, from the "NCBI GEO database" (Home - GEO - NCBI) and previous studies.

\section{Differentially Methylated CpG (DMC) region of chosen genes and designing primers}

We investigated the regulator regions from the "GeneCards database" (GeneCards - Human Genes | Gene Database | Gene Search) to identify the promoter regions. The "UCSC Genome Browser" (UCSC Genome Browser Home) was used to gain the promoters' sequences. The promoters' methylation assessment was utilized by "CpGPlot/CpGreport" (EMBOSS Cpgplot < Sequence Statistics < EMBL-EBI). We considered CpG islands with a high-frequency of $\mathrm{CpG}$ dinucleotides, the $\mathrm{Obs} / \mathrm{Exp}$ value is greater than 0.6 , and the $\mathrm{GC}$ content is higher than $50 \%$ characteristics. (Fig. 1)

Afterward, the restriction endonuclease recognition sites are determined by the "NEBcutter analysis tool" (NEBcutter V2.0), and the specific methylation-sensitive restriction enzymes have been chosen. Then, primer sequences are designed by "Primer3Plus" (https://primer3plus.com/) on either side of the selected restriction enzyme recognition sites. Designed Primers for amplifying the selected genes with methyl sensitive restriction enzyme polymerase chain reaction (MSRE-PCR) method are shown in Table 2. 
Table 2

Primer sequences for DOK7 and SPDEF, their product sizes, methyl-sensitive restriction enzymes, enzyme recognition sites, and their related methylated region. F: Forward. R: Reverse.

\begin{tabular}{|llllll|}
\hline Gene & Primer Sequence $\left(5^{\prime}-\mathbf{3}^{\prime}\right)$ & $\begin{array}{l}\text { Product } \\
\text { Size }\end{array}$ & $\begin{array}{l}\text { Restriction } \\
\text { Enzyme }\end{array}$ & $\begin{array}{l}\text { Cutting } \\
\text { Site }\end{array}$ & $\begin{array}{l}\text { Methylation } \\
\text { Sensitivity }\end{array}$ \\
\hline DOK7 & F: TAGCGACACGGGTTACCGA & $304 \mathrm{bp}$ & Smal & & CpG: blocked \\
& R: TTCCACAAGCACAGCTCAACC & & & & \\
SPDEF & $\begin{array}{l}\text { F: } \\
\text { CCTCAGCTGATCCATCTTAGAAC } \\
\text { R: AGCCGCGAGATGAAGAGTT }\end{array}$ & $302 \mathrm{bp}$ & Hhal & & \\
\hline
\end{tabular}

\section{Study design and participants}

In this study, 95 samples were analyzed, including 30 patients with gastric cancer and 30 patients with intestinal metaplasia, and 35 normal control samples by healthy individuals. All patients sign the consent form to use their clinical samples under the supervision of their physician. This study has been confirmed by the ethical committee of the National Institute of Genetic Engineering and Biotechnology with the code number IR.NIGEB.EC1398.12.3.A. About $4 \mathrm{~mL}$ of whole blood samples were collected in EDTA tubes from participants. The participant's clinicopathological features are presented in Table 1, including age, sex, and the disease stage.

Table 1

Demographic and pathologic data for studied participants

\begin{tabular}{|c|c|c|c|}
\hline \multicolumn{3}{|l|}{ Factors } & Patients $(n=95)$ \\
\hline \multirow[t]{2}{*}{ Age } & \multicolumn{2}{|c|}{ Mean age (year) \pm Std. Deviation } & $60.54 \pm 11.096$ \\
\hline & \multicolumn{2}{|c|}{ Minimum - Maximum } & $27-82$ \\
\hline \multirow[t]{2}{*}{ Gender } & \multicolumn{2}{|l|}{ Female } & $45(47.4 \%)$ \\
\hline & \multicolumn{2}{|l|}{ Male } & $50(52.6 \%)$ \\
\hline \multirow[t]{6}{*}{ Diagnosis } & \multicolumn{2}{|c|}{ Intestinal Metaplasia } & $30(31.6 \%)$ \\
\hline & \multirow[t]{4}{*}{ Cancer } & Stage I & $1(1.1 \%)$ \\
\hline & & Stage II & $8(8.4 \%)$ \\
\hline & & Stage III & $13(13.7 \%)$ \\
\hline & & Stage IV & $8(8.4 \%)$ \\
\hline & \multicolumn{2}{|l|}{ Normal } & $35(36.8 \%)$ \\
\hline
\end{tabular}

Genomic DNA isolation 
DNA samples were extracted by using the GeneAll Biotechnology DNA extraction Kit (GeneAll Biotechnology, Korea) and stored at $-20^{\circ} \mathrm{C}$. The quality and quantity of DNA were determined by The NanoDrop spectrophotometer (Thermo Scientific, Waltham, MA, USA). Also, extracted DNAs run on the $1 \%$ agarose gel to evaluate the quality of their extraction.

\section{Analysis of promoter methylation with MSRE-PCR}

Promoter's CpG islands methylation changes carried out using the MSRE-PCR method. According to the manufacturer's instruction, extracted DNA was digested using the appropriate methylation-sensitive restriction enzyme (RE), Smal for DOK7, and Hhal for SPDEF (Takara, Japan) (Table 2). 50 ng of DNA treated with $0.5 \mu \mathrm{L}$ of RE, $1 \mu \mathrm{L} 10 \times$ universal buffer, sterile distilled water to reach the solutions' total volume to $10 \mu \mathrm{L}$. For undigested samples (as controls), $0.5 \mu \mathrm{L}$ sterile distilled water was added instead of restriction enzyme. All sample tubes were incubated overnight at $37^{\circ} \mathrm{C}$.

Each PCR reaction consisted of $1 \mu \mathrm{L}$ of the DNA solution, $0.5 \mu \mathrm{L}$ of each forward and reverse primer (with the concentration of $10 \mathrm{pmol} / \mu \mathrm{L}$ ), and $10 \mu \mathrm{L}$ of Taq $2 \times$ master mix, sterile distilled water to achieve a 20 $\mu \mathrm{L}$ reaction mixture for each sample. Treated DNA amplified by PCR using DOK7- and SPDEF-specific primers (Table 2) and the Taq PCR Master Mix Kit (Ampliqon, Denmark).

PCR condition was performed in 30 cycles, including strings denaturation $\left(95^{\circ} \mathrm{C}\right.$ for $\left.40 "\right)$, primer annealing $\left(60^{\circ} \mathrm{C}\right.$ for $\left.40^{\prime \prime}\right)$, extension $\left(72^{\circ} \mathrm{C}\right)$. Also, initial denaturation set at $95^{\circ} \mathrm{C}$ for $5^{\prime}$, and a final extension set at $72^{\circ} \mathrm{C}$ for 7 ' were considered. PCR products run on the $1.5 \%$ agarose gel, stained with ethidium bromide and bands visualized under UV radiation.

To identify the accurate samples methylation, each treated sample with RE was compared with its undigested one as $100 \%$ methylated due to no RE were used in these tubes. The intensity of the treated samples amplified products has a direct relationship with the methylation level. The methylation intensity was calculated by gel analyzer software (GelAnalyzer 19.1).

\section{Quantifying with MSRE-qPCR}

Real-time PCR was applied to quantify methylation alteration in DOK7 and SPDEF genes. For each sample, digested and undigested DNA samples were amplified. All PCRs were performed in a Rotor-Gene 6000 thermal cycler (Corbett Life Science, Australia). Real-time PCR performed with the following constituents: $1 \mu \mathrm{L}$ of DNA solution was added to $9 \mu \mathrm{L}$ of a PCR mixture made up of $5 \mu \mathrm{L}$ of $2 \times$ SYBR Green PCR Master Mix (Takara, Japan), $0.2 \mu \mathrm{L}$ of forward and reverse primers, and $4.6 \mu \mathrm{L}$ of water. PCR condition was performed in 45 cycles, including strings denaturation $\left(95^{\circ} \mathrm{C}\right.$ for $\left.30^{\prime \prime}\right)$, primer annealing $\left(60^{\circ} \mathrm{C}\right.$ for $\left.30^{\prime \prime}\right)$, extension $\left(72^{\circ} \mathrm{C}\right.$ for $\left.30^{\prime \prime}\right)$. Also, initial denaturation is set at $95^{\circ} \mathrm{C}$ for $5^{\prime}$.

$\triangle \mathrm{Ct}$ values determined as the difference between the obtained $\mathrm{Ct}$ values of the non-treated DNA and treated DNA $\left(\Delta \Delta C_{T}=\Delta C_{T(U N)}-\Delta C_{T(D)}\right)$.

\section{Statistical analysis}


Results expressed as means \pm standard deviation (SD), and all statistical analyses were performed using IBM SPSS version 26 (SPSS, Inc., Chicago, IL, USA) and Prism (GraphPad Software, San Diego, CA) version 9.0.0. The statistical significance of the difference between groups was determined using oneway ANOVA to determine the three groups' differences. $\mathrm{P}<0.05$ were considered as statistically significant.

\section{Results}

\section{Clinicopathological parameters}

The present study analyzed the association between the promoter methylation status of DOK7 and SPDEF genes and clinicopathological characteristics. The patient's age range was between $27-78$ years old, 41-81 years old, and 37-82 years old for normal, metaplasia, and gastric cancer cohorts, respectively. Also, $47.4 \%$ and $52.6 \%$ of patients were female and male. (Table 1 )

\section{Differences in methylation between normal, metaplasia, and gastric cancer specimens by MSRE-PCR method}

In this study, the relationship between methylation patterns of DOK7 and SPDEF promoters of 60 patients with metaplasia or gastric cancer and 35 normal individuals were analyzed. Our assessments indicate that the DOK7 promoter was hypomethylated in normal samples (mean $=57.77 \%$ ) compared with IM (mean $=74 \%$ ) and GC (mean $=88.07 \%$ ) patients. Also, the methylation of the SPDEF promoter was lower in normal individuals $($ mean $=54.43 \%)$ than GC (mean $=81.47 \%)$ and IM (mean $=63.13 \%)($ Table 3$)$. 
Table 3

The percentage of DOK7 and SPDEF promoters hypermethylation status after analysis of $\mathrm{Ct}$ obtained from real-time PCR test. The results of normality tests have demonstrated that methylation status has a normal distribution. IM: Intestinal Metaplasia, GC: Gastric Cancer.

\begin{tabular}{|lllllll|}
\hline Genes & DOK7 & \multicolumn{5}{c|}{ SPDEF } \\
\hline Factor (s) & & & & & & \\
\hline Patients & Normal & IM & GC & Normal & IM & GC \\
\hline Minimum (\%) & 25 & 35 & 65 & 25 & 40 & 50 \\
\hline Maximum (\%) & 85 & 100 & 100 & 90 & 100 & 100 \\
\hline Range (\%) & 60 & 65 & 35. & 65 & 60 & 50 \\
\hline Mean (\%) & 56.8 & 66 & 88.1 & 58.8 & 71.3 & 81.5 \\
\hline Probability normal (Gaussian) & $70.1 \%$ & $83.7 \%$ & $85.3 \%$ & $77.6 \%$ & $80.5 \%$ & $89 \%$ \\
\hline Lower 95\% Cl of mean & 51 & 59.7 & 84.5 & 53.1 & 65.05 & 75.8 \\
\hline Upper 95\% Cl of mean & 62.5 & 72.3 & 91.7 & 64.4 & 77.6 & 87.1 \\
\hline & & & & & & \\
\hline
\end{tabular}

The difference between the methylation percentage of the DOK7 promoter in normal versus metaplasia and gastric cancer samples were significant ( $p$-value $<0.001$ ). Also, the difference in SPDEF promoter methylation in normal samples compared to metaplasia and gastric cancer patients was significant ( $p$ value $<0.001$ ). (Fig. 2, Fig. 3)

The comparison of methylated DOK7 levels in GC patients and normal controls ( $p$-value $<0.001$ ) revealed high intermediate sensitivity, specificity $73.33,97.14 \%$, respectively, with a cut-off at $>84.50 \%$ and area under the ROC curve of 0.9495. Also, the examination of methylated SPDEF ( $p$-value $<0.001$ ) revealed low sensitivity and high specificity values of 36.67 and $97.14 \%$, respectively, with a cut-off at $>89.5$ and an area under the ROC curve of 0.8419 (Fig. 4).

\section{Discussion}

In this study, alterations of DOK7 and SPDEF promoter methylations were examined in DNA extracted from whole blood samples of $30 \mathrm{GC}$ and $30 \mathrm{IM}$ cases compared to 35 normal controls. Significant methylation changes were observed between cases (GC and IM) and controls. For Dok7 the level of significances were GC ( $<<0.001), I M(p=0.03)$ and for SPDEF they were GC $(p<0.001)$ and IM $(p=0.03)$ respectively. Interestingly, significant differences also were observed between the methylation status of GCs and IM cases themselves for DOK7 $(p<0.001)$ and for SPDEF $(p=0.03)$. 
Our results conveyed that the mean of methylation in the DOK7 promoter was equal to $66 \%$ in DNA extracted from WBCs in IM cases, whereas it was $88.07 \%$. In GC cases, and demonstrated significantly $22 \%$ less methylation compared to IM cases. The significant results also were detected for SPDEF, in which more than $10 \%$ methylation status was observed in GCs (mean $81.47 \%$ ) compared to IM (mean $71.33 \%)$ cases.

In IM patients, the methylation status difference with normal controls was $9.17 \%$ for DOK7 and $12.56 \%$ for SPDEF. These results suggest that the methylation status is significantly elevated towards hypermethylation of DOK7 and SPDEF from normal controls to IMs and GCs, respectively.

The same results have been reported previously for DOK7 promoter methylation changes in tumor samples from breast cancer [16], esophageal squamous cell carcinoma [17], and glioma [18], in which hypermethylation of DOK7 was reported in those cancers. Moreover, a significant reduction in DOK7 expression has been shown in lung cancer patients, also associated with poor survival [13]. Significant hypermethylation of SPDEF has been reported in lung metaplasia, chronic obstructive pulmonary disease (COPD) [19].

DNA methylation changes, including local hypermethylation and global (genome-wide) hypomethylation, are associated with cancer development and tumor growth [20]. DNA hypermethylation, rationally, is not a random process and can accurately characterize the cancer status. If selected DNA methylation markers are consistent and replicable, they can be utilized in clinical practices. Since blood is a convenient tissue in noninvasive cancer assays, evaluation of cancer biomarker is under intensive attention in DNA extracted from WBC [21].

The exosomes are cell to cell communication particles secreted from a different cell type consisting of immune and cancerous cells. They contain malignant information such as nucleic acids, mRNAs or miRNAs, or proteins, such as growth factors, cytokines, chemokines, or angiogenic and immunoregulatory molecules in their cargo. These cancer-released exosomes can induce tumor growth, neovascularization, immune suppression, invasion, dissemination, and pre-metastatic niche formation. They have an important role in cancer development and metastasis [22]. Boonsongserm and colleagues demonstrated that colorectal cancer (CRC) cell exosomes induce genome-wide DNA methylation changes in the WBCs of patients with CRC [23]. Besides, several studies reported global changes in repetitive elements [24], as well as specific $\mathrm{CpG}$ methylation alterations found in DNA from blood cells of patients with various cancer types $[25,26]$.

ROC analysis was employed to compare the predictive accuracy of the methylation status of patients with gastric cancer. Our findings demonstrated the methylation status of DOK7 (with a cut-off at $>84.50$ $\%)$ could reflect the gastric lesions' malignancy.

In conclusion, this study is the first report that revealed a significant alteration in DOK7 and SPDEF promoter's methylation observed in the WBCs of patients with either IM or GC as a promising tumor marker for cancer screening studies. 


\section{Conclusions}

These results suggest that the change in the methylation of DOK7 $(A U C=0.9495)$ and SPDEF $(A U C=$ 0.8419) is a promising epi-biomarker. For the first time, this study shows blood-based biomarkers DOK7 and SPDEF genes are powerful epi-biomarkers and provide insights into gastric cancer pathogenesis and diagnosis.

\section{Declarations}

\section{Ethics approval and Consent to participation}

The study was approved by the Ethical Committee of the National Institute of Genetic Engineering and Biotechnology (NIGEB) (Ethical code \#: IR. NIGEB.EC1398.12.3.A). The informed consent form has been prepared and signed by all of the participants and the parents of the minors under eighteen years old to use their clinical samples and personal data under the supervision of their physician. All methods were carried out in accordance with relevant guidelines and regulations.

\section{Availability of data and materials}

The datasets generated and/or analyzed during the current study have uploaded in the NCBI GEO repository, "GEO Accession viewer." 29 May. 2020, https://www.ncbi.nlm.nih.gov/geo/query/acc.cgi? acc=GSE30601.

\section{Consent for publication}

Not applicable.

\section{Competing interests}

All authors have read the manuscript and declared that they have no competing interest.

\section{Authors' contributions}

The concepts and methodology were designed by SAA. The sampling and experimental laboratory work were carried out by AM and MR. The data acquisition, analyzing and interpretation were performed by AM and SAA. The manuscript writing and revisions were performed by AM and SAA. Administrative, technical, and material were supported by SAA, KM, AA, BA. This study was supervised by SAA. All authors read and approved the final manuscript.

\section{Funding}

Our study was supported by grants number 633 and 667 from the National Institute of Genetic Engineering and Biotechnology (NIGEB). 


\section{Acknowledgment}

Work was carried out at the National Institute of Genetic Engineering and Biotechnology (NIGEB), and the authors would like to express their appreciation to NIGEB.

\section{References}

1. Siegel, R.L., K.D. Miller, and A. Jemal, Cancer statistics, 2020. CA: A Cancer Journal for Clinicians, 2020. 70(1): p. 7-30.

2. Liu, K.S., I.O. Wong, and W.K. Leung, Helicobacter pylori associated gastric intestinal metaplasia: Treatment and surveillance. World J Gastroenterol, 2016. 22(3): p. 1311-20.

3. Leiting, J.L. and T.E. Grotz, Advancements and challenges in treating advanced gastric cancer in the West. World J Gastrointest Oncol, 2019. 11(9): p. 652-664.

4. McLean, M.H. and E.M. El-Omar, Genetics of gastric cancer. Nature Reviews Gastroenterology \& Hepatology, 2014. 11(11): p. 664-674.

5. Meyer, A.R. and J.R. Goldenring, Injury, repair, inflammation and metaplasia in the stomach. The Journal of physiology, 2018. 596(17): p. 3861-3867.

6. Shao, L., et al., Risk of gastric cancer among patients with gastric intestinal metaplasia. Int J Cancer, 2018. 143(7): p. 1671-1677.

7. Sitarz, R., et al., Gastric cancer: epidemiology, prevention, classification, and treatment. Cancer Manag Res, 2018. 10: p. 239-248.

8. Chen, K., et al., Mutational landscape of gastric adenocarcinoma in Chinese: implications for prognosis and therapy. Proceedings of the National Academy of Sciences of the United States of America, 2015. 112(4): p. 1107-1112.

9. Gigek, C.O., et al., Epigenetic mechanisms in gastric cancer. Epigenomics, 2012. 4(3): p. 279-294.

10. Agha, G., et al., Blood Leukocyte DNA Methylation Predicts Risk of Future Myocardial Infarction and Coronary Heart Disease. Circulation, 2019. 140(8): p. 645-657.

11. Buyan, A., A.C. Kalli, and M.S.P. Sansom, Multiscale Simulations Suggest a Mechanism for the Association of the Dok7 PH Domain with PIP-Containing Membranes. PLoS computational biology, 2016. 12(7): p. e1005028-e1005028.

12. Fitzgerald, L.M., et al., ELF5 and DOK7 regulation in anti-estrogen treated cells and tumors. Cancer Cell Int, 2016. 16: p. 8.

13. Chen, G., et al., The downstream of tyrosine kinase 7 is reduced in lung cancer and is associated with poor survival of patients with lung cancer. Oncol Rep, 2017. 37(5): p. 2695-2701.

14. Lo, Y.H., et al., Transcriptional Regulation by ATOH1 and its Target SPDEF in the Intestine. Cell Mol Gastroenterol Hepatol, 2017. 3(1): p. 51-71.

15. Wu, J., et al., SPDEF is overexpressed in gastric cancer and triggers cell proliferation by forming a positive regulation loop with FoxM1. J Cell Biochem, 2018. 119(11): p. 9042-9054. 
16. Heyn, H., et al., DNA methylation profiling in breast cancer discordant identical twins identifies DOK7 as novel epigenetic biomarker. Carcinogenesis, 2013. 34(1): p. 102-8.

17. Yang, S.M., et al., Repression of DOK7 mediated by DNMT3A promotes the proliferation and invasion of KYSE410 and TE-12 ESCC cells. Biomed Pharmacother, 2017. 90: p. 93-99.

18. Hua, C.D., et al., Repression of Dok7 expression mediated by DNMT1 promotes glioma cells proliferation. Biomed Pharmacother, 2018. 106: p. 678-685.

19. Song, J., et al., Aberrant DNA methylation and expression of SPDEF and FOXA2 in airway epithelium of patients with COPD. Clinical Epigenetics, 2017. 9(1): p. 42.

20. Tahara, T. and T. Arisawa, DNA methylation as a molecular biomarker in gastric cancer. Epigenomics, 2015. 7(3): p. 475-86.

21. Tahara, T., et al., Methylation status of IGF2 DMR and LINE1 in leukocyte DNA provides distinct clinicopathological features of gastric cancer patients. Clin Exp Med, 2018. 18(2): p. 215-220.

22. Ferguson Bennit, H.R., et al., Peripheral Blood Cell Interactions of Cancer-Derived Exosomes Affect Immune Function. Cancer microenvironment: official journal of the International Cancer Microenvironment Society, 2019. 12(1): p. 29-35.

23. Boonsongserm, P., et al., Tumor-induced DNA methylation in the white blood cells of patients with colorectal cancer. Oncol Lett, 2019. 18(3): p. 3039-3048.

24. Puttipanyalears, C., et al., Breast cancer upregulating genes in stromal cells by LINE-1 hypermethylation and micrometastatic detection. Epigenomics, 2016. 8(4): p. 475-86.

25. Brennan, K., et al., Intragenic ATM Methylation in Peripheral Blood DNA as a Biomarker of Breast Cancer Risk. Cancer Research, 2012. 72(9): p. 2304.

26. Chopra-Tandon, N., et al., Relationships between Global DNA Methylation in Circulating White Blood Cells and Breast Cancer Risk Factors. Journal of Cancer Epidemiology, 2017. 2017: p. 2705860.

\section{Figures}




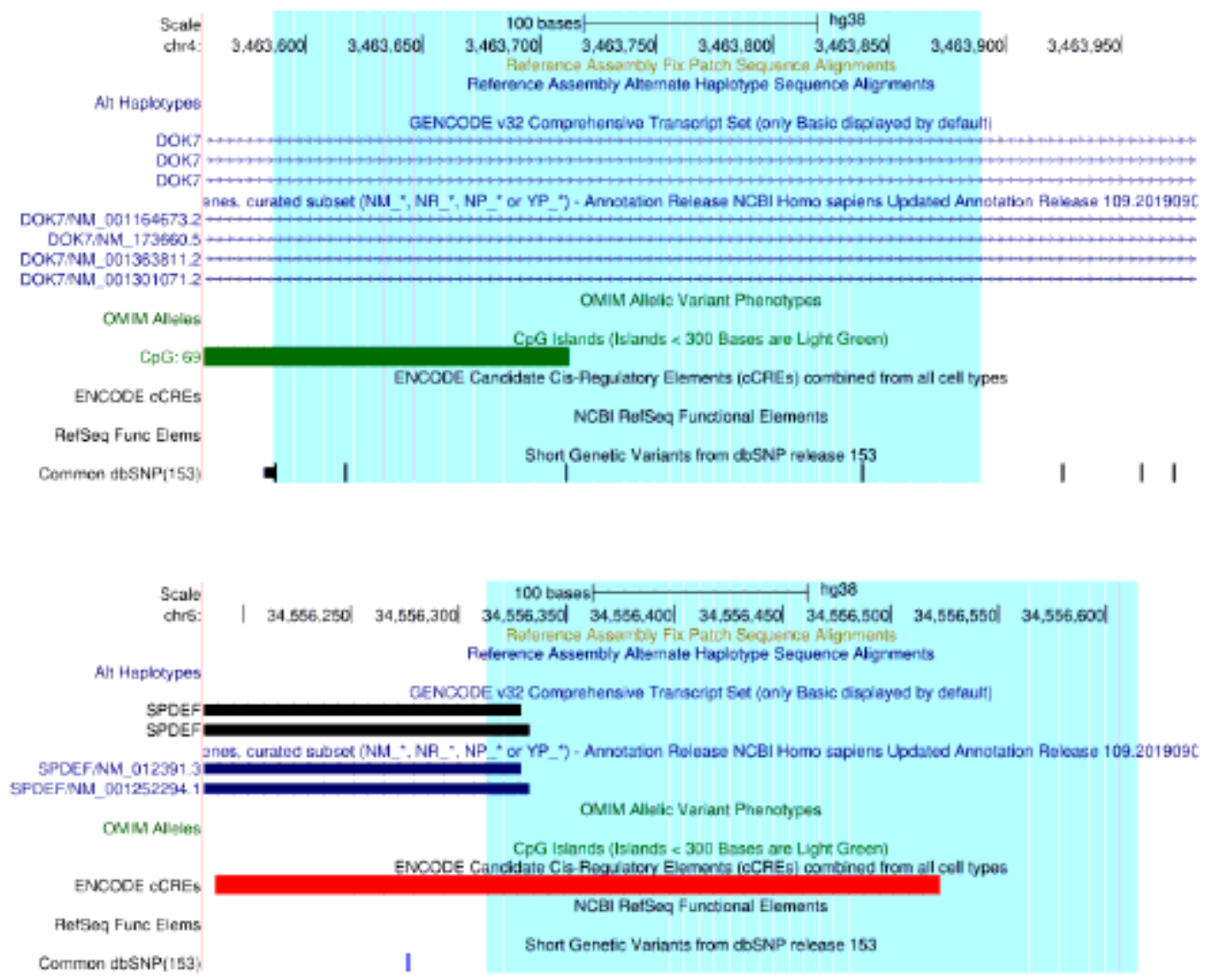

Figure 1. Pictures are adapted from the UCSC genome browser image - for the genomic location of $\mathrm{CpG}$. The cyan highlights are our additions gained from the $\mathrm{CpG}$ plot and highlight our desired genomic regions to amplify. Upper and lower figures represent desired genomic regions for DOK 7 and SPDEF, respectively.

\section{Figure 1}

Pictures are adapted from the UCSC genome browser image - for the genomic location of CpGs. The cyan highlights are our additions gained from the $\mathrm{CpG}$ plot and highlight our desired genomic regions to amplify. Upper and lower figures represent desired genomic regions for DOK7 and SPDEF, respectively.

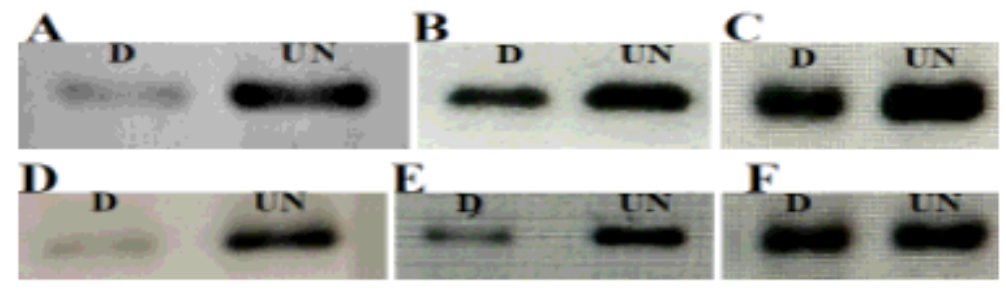

Figure 2. Methylation status of DOK7 and SPDEF after digestion and MS-PCR of DNA samples treated (D) smaple compared untreated (UN) control. Upper lin (A) Normal (B) Metaplasia (C) Gastric cancer for DOK7. Lower line (D) Normal (E) Metaplasia (F) Gastric cancer for SPDEF. 
Figure 2

Methylation status of DOK7 and SPDEF after digestion and MS-PCR of DNA samples treated (D) smaple compared untreated (UN) control. Upper lin (A) Normal (B) Metaplasia (C) Gastric cancer for DOK7. Lower line (D) Normal (E) Metaplasia (F) Gastric cancer for SPDEF.
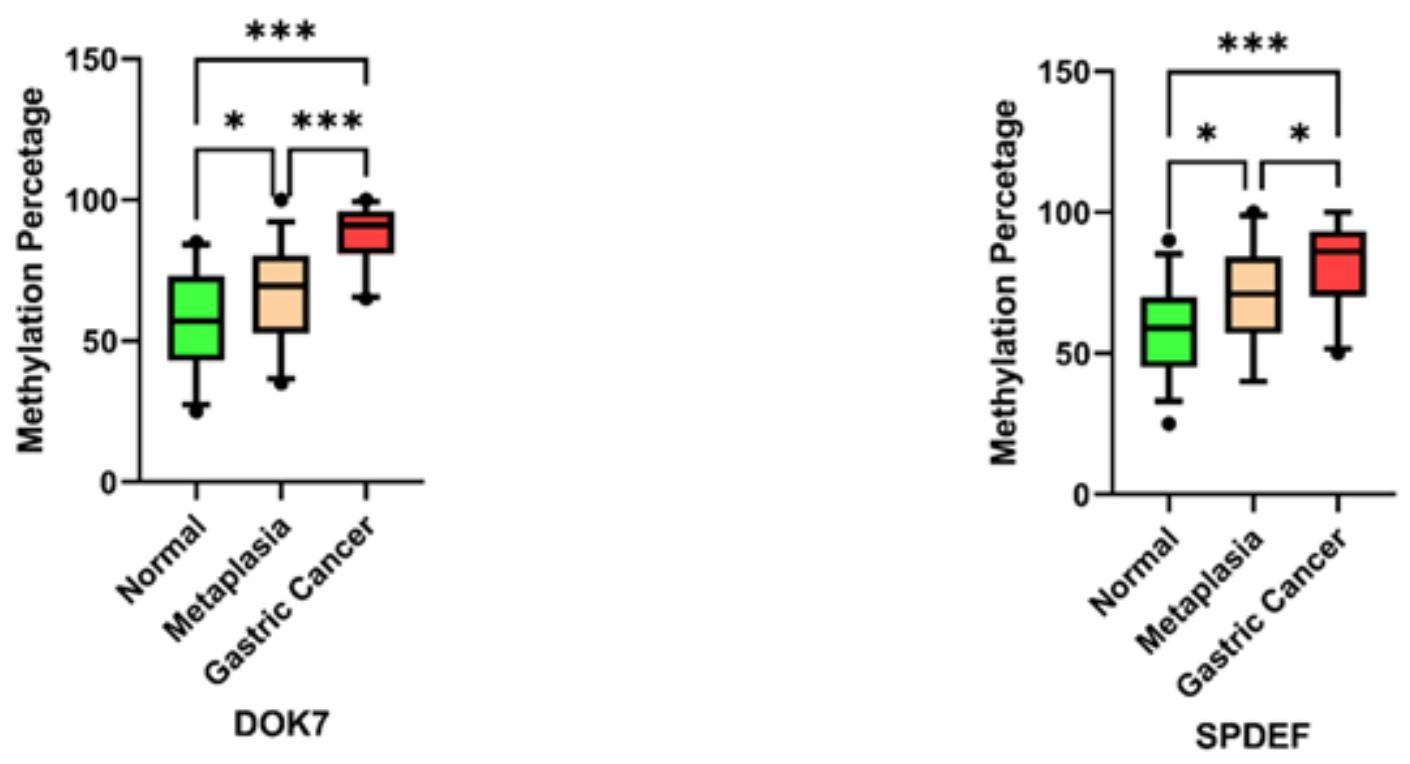

Figure 3. Dok7 and SPDEF methylation status in the DNA obtained from whole blood. Increased methylation of DOK7 and SPDEF promoters correlated with the presence of various gastric lesions. Each bar represents the mean $\pm S D$. ( $p$-value $=0.05$ considered, ${ }^{*}: p=0.03,{ }^{* * *} p<0.001$ vs. normal control)

\section{Figure 3}

Dok7 and SPDEF methylation status in the DNA obtained from whole blood. Increased methylation of DOK7 and SPDEF promoters correlated with the presence of various gastric lesions. Each bar represents the mean \pm SD. ( $p$-value $=0.05$ considered, $*: p=0.03$, $* \star \star ~ p<0.001$ vs. normal control) 

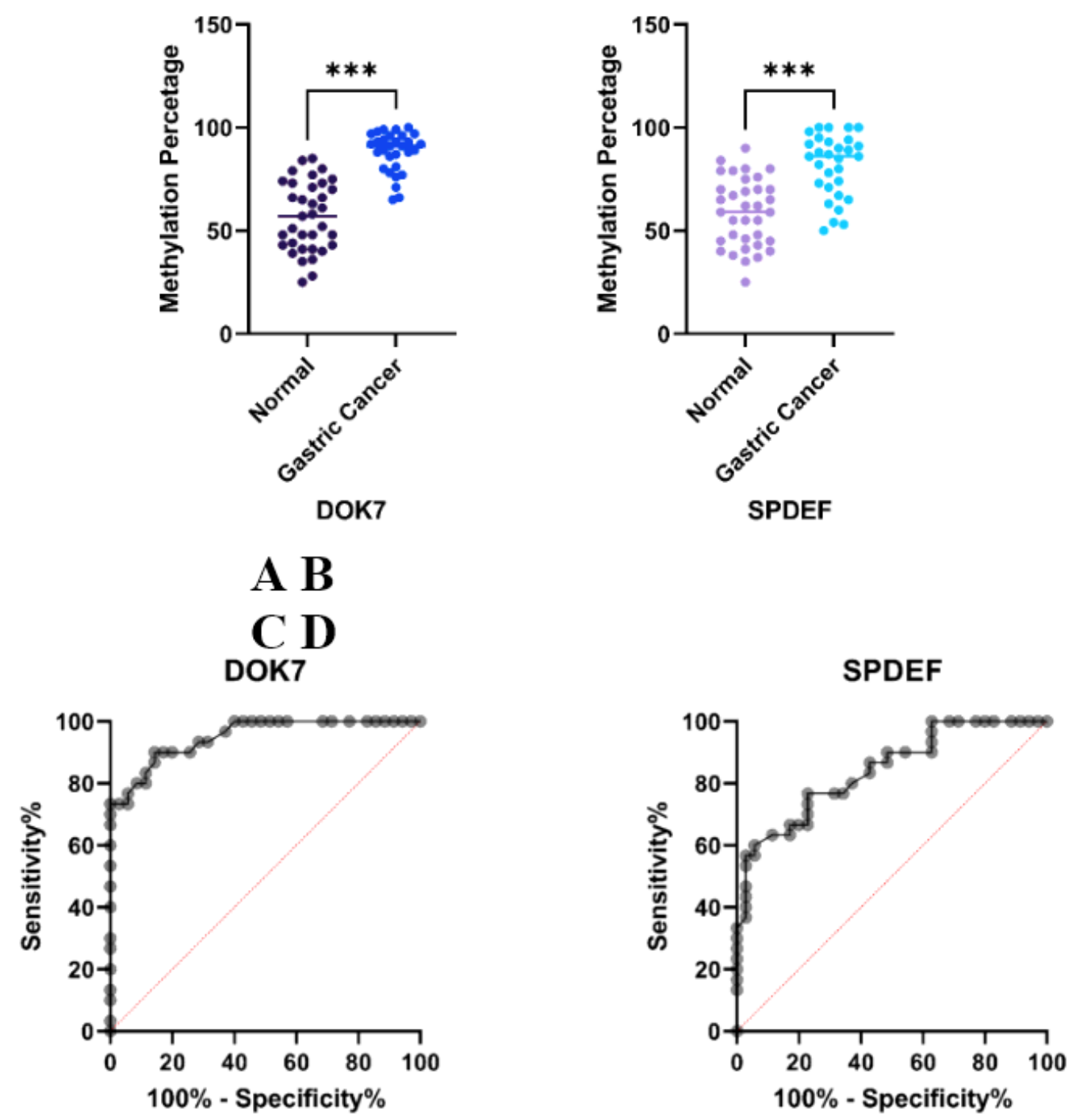

Figure 4. Methylation status in WBCs determined by qMSRE-PCR. (A) Scatter plot of DOK7 methylation between 30 patients with $\mathrm{GC}$ and 35 normal controls. The levels of DOK methylation are expressed as the amount for each sample and mean. (*** p < 0.001). (B) Scatter plot of SPDEF methylation between $30 \mathrm{GC}$ patients and 35 normal controls. The levels of SPDEF methylation are expressed as the amount for each sample and mean. (** $\mathrm{p}<0.001$ ). (C) ROC curve of DOK 7 methylation $(\mathrm{p}<0.001)$ exhibiting a well-validated gene with high intermediate sensitivity (73.33\%) and high specificity (97.14\%). (D) ROC curve of SPDEF methylation $(\mathrm{p}<0.001)$ showing a defective validated gene with low sensitivity $(36.67 \%)$ but high specificity (97.14\%).

\section{Figure 4}

Methylation status in WBCs determined by qMSRE-PCR. (A) Scatter plot of DOK7 methylation between 30 patients with GC and 35 normal controls. The levels of DOK methylation are expressed as the amount for each sample and mean. ( $\star \star \star ~ p<0.001)$. (B) Scatter plot of SPDEF methylation between $30 \mathrm{GC}$ patients and 35 normal controls. The levels of SPDEF methylation are expressed as the amount for each sample and mean. (*** $p<0.001)$. (C) ROC curve of DOK7 methylation $(p<0.001)$ exhibiting a well-validated 
gene with high intermediate sensitivity (73.33 \%) and high specificity (97.14 \%). (D) ROC curve of SPDEF methylation $(p<0.001)$ showing a defective validated gene with low sensitivity $(36.67 \%)$ but high specificity $(97.14 \%)$. 